\title{
Morbidity using subcutaneous ports and efficacy of vancomycin flushing in cancer
}

H Rubie, M Juricic, S Claeyssens, A Krimou, J Lemozy, P Izard, J Guitard, M Ane, M F Prere, F Fedacou, A Robert, C Regnier
Abstract

An evaluation of totally implanted venous access systems inserted in 163 consecutive children with cancer is reported. From 1988 to 1994,180 subcutaneous ports were inserted in children more than 1 year old. Initial diagnosis was acute leukaemia $(\mathbf{n}=79)$, non-Hodgkin's lymphoma $(\mathbf{n}=33)$, and solid tumour $(n=51)$. Median age was 85 months. All venous procedures were performed through the device. Chemotherapy was either moderate $(n=13)$ or intensive $(n=119)$ or very intensive $(n=48)$, including 16 patients undergoing marrow transplantation. Cumulative venous access totalled 55770 patient days with a mean of 305 days/subcutaneous port. The cause of device removal was, end of treatment $(n=111)$, death due to malignancy $(n=20)$, catheter related infection $(n=7)$, and occlusion of the system $(n=4)$. Mechanical complications occurred in 19 ports; 16 were due to clots, of which 14 were cleared with instillation of urokinase. Documented infectious episodes occurred in 47 ports, recurred once in 14, and twice in five cases. Among these infections, 47 were septicaemic; 31 due to Staphylococcus epidermidis. Twenty seven of initial septic episodes were considered to be catheter related; the rate was $15 \% /$ subcutaneous port or $0 \cdot 05 / 100$ catheter days. Risk factors for the development of a first infection were age below 4 years and the time of use. Since February 1993, vancomycin (50 $\mu \mathrm{g} / \mathrm{ml}$ ) has been given and this has reduced the rate of $S$ epidermidis infection from 26/83 subcutaneous port to 4/97. Life table analysis showed that the infection free interval for staphylococcus was significantly better after this technique was initiated $(\log$ rank test $=0 \cdot 02)$. Time saved was approximately 30minutes/patient/ week compared with external catheters, or 45 hours/month for the cohort of children treated. Subcutaneous ports in paediatric cancer patients are reliable, safe, and durable and may offer an attractive alternative to external catheters for prolonged venous access and intensive treatment.

(Arch Dis Child 1995; 72: 325-329)

Keywords: ports, cancer, vancomycin flushing.

Correspondence and reprint requests to: Dr H Rubie, Unit of Paediatric Haematology and Oncology, Service de Médecine Infantile B, CHU Purpan 31059 Toulouse Cédex France.

Accepted 22 November 1994

Venous access is one of the basic challenges in treating children with malignancies. The patients need frequent blood sampling, administration of antineoplastic drugs, and may additionally require intravenous fluids, antibiotics, and blood products as well as parenteral nutrition. The use of indwelling central venous catheters has been customary in the past because it has simplified patient care with greater comfort. ${ }^{12}$ However, the need for frequent care to conserve them and the risk of infection are major limiting factors. ${ }^{3-5}$ In recent years, totally implanted subcutaneous venous access systems have been used successfully in adult patients with cancer. ${ }^{67}$ Experience is limited in children ${ }^{8-11}$ and only a few studies have compared external and subcutaneous devices in children with cancer but they suggest a lower incidence of infections and better comfort with implanted ports. ${ }^{12} 13$

We report an evaluation of the use of subcutaneous ports in 163 consecutive children with malignancy in a single institution and the impact of a vancomycin flush technique to decrease the incidence of catheter related infection.

\section{Patients and methods}

PATIENT SELECTION

From April 1988 to October 1994, 163 consecutive haematology/oncology patients were selected to receive a subcutaneous port because they needed chemotherapy and it was likely that repeated venepuncture would be necessary. Patients who were to receive light chemotherapy for disorders such as a low stage of Hodgkin's disease or nephroblastoma were not considered candidates for either central venous access or a port. Infants or children with body weights below $10 \mathrm{~kg}$ received external catheters. Patients requiring intensive treatment, including high dose chemotherapy followed by bone marrow transplantation (BMT), were selected as the primary candidates for a subcutaneous port, except in the allogeneic setting. The ports were removed in most patients at the end of chemotherapy protocols but were maintained for a time in some patients with high risk of relapse.

LINE INSERTION AND CARE TECHNIQUES Implantation procedure

Before the insertion of a subcutaneous port, anaemia (a haemoglobin concentration $<80 \mathrm{~g} / \mathrm{l}$ ) and/or thrombocytopenia (a platelet count $<50 \times 10^{9} 1$ ) were corrected by blood transfusions and electrolyte abnormalities were All lines were inserted in the operating theatre, under general anaesthesia and sterile 
conditions, by the same surgical team. The catheters (small titanium port with preconnected radio-opaque silicone venous catheter, 6.6 French, 1.0 lumen; Hickman II Bard) were tunnelled subcutaneously in the chest wall and inserted, preferably, in the right external jugular vein; if the right external jugular vein was too small, absent, or occluded because of previous catheter placement, the ipsilateral internal jugular or the contralateral external jugular was used. Under scope guidance, the catheter was placed in the superior vena cava. A pocket was made under the subcutaneous tissue to accommodate the port. Postoperatively, the system was flushed with saline containing $100 \mathrm{U} / \mathrm{ml}$ heparin. Subcutaneous absorbable sutures were used to close the incision with reinforcement by Steristrips. A chest radiograph was always done for initial reference. A subcutaneous port was not used until 48 hours after surgery except in some cases where specific treatment could not be begun via a peripheral vein.

\section{Catheter used and care}

Access to the system was achieved using the same sterile techniques applicable to standard venous catheters. The overlying skin was cleaned with iodophore solution. The unit was flushed with saline before infusions and with saline containing $100 \mathrm{U} / \mathrm{ml}$ heparin either daily or after every blood sampling procedure. A 22 gauge right angle non-coring needle, bent at $90^{\circ}$, was used for infusions. The needle was secured with sterile gauze and adhesive dressing. We have used Gripper needles (Pharmacia) since early 1991. The needle and the dressing were changed every five days. For outpatients, the port was flushed monthly with $3-5 \mathrm{ml}$ of diluted heparin solution $(100 \mathrm{U} / \mathrm{ml})$. External catheters were maintained using the same procedures of heparinised flushes and dressings except in outpatients where maintenance was required every week. There were many instances of infections with Staphylococcus epidermidis in ports implanted before 1 February 1993, so we instituted the practice of adding vancomycin $(50 \mu \mathrm{g} / \mathrm{ml})$ to the flushing solution.

Blood was withdrawn, and all medications (chemotherapeutic agents, transfusions, parenteral nutrition, antibiotics, analgesics, antiemetics, etc) were administered through the system.

Chemotherapy was defined as 'moderate' if the total duration of neutropenia was under 30 days, and/or if the system was used less than once a week. Chemotherapy was considered as 'very intensive' if neutropenia lasted more than 100 days, and/or if more than five procedures/day were performed, or if the patient underwent BMT. In all other cases, chemotherapy was considered as 'intensive'.

\section{Complications}

Infection was defined as local if underlying skin only was infected and the access to the port
Table 1 Characteristics of the 180 subcutaneous ports

\begin{tabular}{lc}
\hline Characteristic & No \\
\hline Diagnosis & \\
Acute lymphoblastic leukaemia & 87 \\
Non-Hodgkin's lymphoma & 33 \\
Acute non-lymphoblastic leukaemia & 7 \\
Solid tumour & 53 \\
Median (range) age (months) & $85(16-219)$ \\
Sex ratio (M/F) & $105 / 75$ \\
Chemotherapy & 13 \\
Moderate & 119 \\
Intensive & $48(16)$ \\
Very intensive (transplantation) & 15 \\
Second catheter & 2 \\
Relapse & $224 / 305(30-1652)$ \\
Infection & 770 \\
Median/mean (range) time of insertion & 34 \\
(days) & 146 \\
Total patient days & 111 \\
Subcutaneous port still in place & 20 \\
Cause of removal & \\
End of treatment & 7 \\
Death due to the disease & 6 \\
Refractory infection related to the & 1 \\
$\quad$ system & 4 \\
Staphylococcus epidermidis & 1 \\
Yeast & 2 \\
Death due to infection & 1 \\
Staphylococcus aureus & 4 \\
Candida & \\
Aspergillus & \\
Mechanical complication & \\
\hline
\end{tabular}

inadvisable. When patients were admitted with fever and neutropenia, blood was obtained for culture exclusively through the port before starting antibiotic treatment. Bacteraemia and septicaemia were defined respectively after obtaining one and at least two positive blood cultures with the same organism during fever. Infections were treated with appropriate antibiotics. Peripheral blood cultures were obtained from venepuncture only when the organism could not be cleared with such antibiotic treatment. The subcutaneous port was removed after local abscess, resistant septicaemia, or fungal septicaemia. Occlusions due to blood clots were treated with urokinase, $1000 \mathrm{U} / \mathrm{ml}$, which was instilled into the system for three hours. When this was unsuccessful, a second urokinase instillation was left in place for up to 24 hours.

\section{STATISTICAL ANALYSIS}

Infections and mechanical complications were expressed as rates. The number of infected lines was expressed as rates $/ 100$ patient days of line placement, as used by most authors. ${ }^{8-12}$ Differences in frequencies of any finding were analysed using the $\chi^{2}$ test with continuity correction. Only $\mathrm{p}$ values $<0.05$ were considered to indicate significance. For sepsis, the time to the outcome was used in a Kaplan-Meier survival analysis, ${ }^{14}$ the survival time being the infection free interval.

\section{Results}

OVERALL POPULATION

Over a six year period, 180 subcutaneous ports were implanted in 163 patients. Their characteristics are summarised in table 1 . During the same period, external catheters were implanted in 50 children because of young age or low weight $(n=19)$ of if indicated by allogeneic BMT $(n=31)$. Neutropenia was noted in all 
Table 2 Characteristics of infectious episodes

\begin{tabular}{|c|c|c|c|}
\hline & $\begin{array}{l}\text { First } \\
\text { infection }\end{array}$ & $\begin{array}{l}\text { Second } \\
\text { infection }\end{array}$ & $\begin{array}{l}\text { Third } \\
\text { infection }\end{array}$ \\
\hline \multicolumn{3}{|l|}{ Delay (days) } & 5 \\
\hline Median & 30 & 97 & 125 \\
\hline Mean & 78 & 140 & 170 \\
\hline Range & $3-5000$ & $26-599$ & 98-333 \\
\hline \multicolumn{4}{|l|}{ Type } \\
\hline Local & $10^{\star}$ & $2^{\star}$ & 1 \\
\hline Bacteraemia & 9 & 4 & 0 \\
\hline Septicaemia & 34 & 9 & 4 \\
\hline \multicolumn{4}{|l|}{ Organism } \\
\hline Staphylococcus epidermidis & $21 \dagger$ & $8 \dagger$ & $2 \dagger$ \\
\hline Staphylococcus aureus & $7^{\circ}$ & 1 & 0 \\
\hline Streptococcus mitis & & 2 & 0 \\
\hline Gram negative & 11 & 2 & 3 \\
\hline Yeast & 4 & 1 & 0 \\
\hline \multicolumn{4}{|l|}{ Outcome } \\
\hline Cure & 38 & 13 & 2 \\
\hline Death from infection & $3 \ddagger$ & $1 \neq$ & 0 \\
\hline Device removal & 4 & 0 & 3 \\
\hline
\end{tabular}

*One bacteraemia, six septicaemia in first infection; one bacteraemia in second infection.

tOne $+S$ aureus, one + Gram negative in first infection; one + Gram negative in second infection; one + Gram negative in third infection.

fOne $S$ aureus + two yeast in first infection; one yeast in second inflection.

patients at some point during the period the port was in place.

\section{INFECTIOUS COMPLICATIONS}

The major complication of the port was infection, occurring with 47 (26\%). Among these patients, 14 developed a second infection and five a third episode of sepsis. Their characteristics are described in table 2. Most were septicaemic (47 out of 66 episodes). $S$ epidermidis accounted for the majority of positive blood cultures (31/61) and constituted the most common first documented infection $(21 / 46)$. Most $(90 \%)$ first infections were cleared with appropriate antibiotic treatment. Infection recurred in 14 children including septicaemia $(n=5)$ and bacteraemia $(n=1)$ with the same organism (S epidermidis) or other organisms $(n=8)$. The subcutaneous port had to be removed because of infection in only seven cases, of whom one had a fungal septicaemia; in six the reason for removal was systemic infection with $S$ epidermidis that was either recurrent $(n=3)$ or refractory $(n=1)$ due to subcutaneous abscess formation $(n=2)$ despite appropriate antibiotic treatment. Four patients died from infection: two had fungal septicaemia (one with Candida parapsilosis and the other Trichosporum cutaneum), one had invasive pulmonary aspergillosis, and one had septicaemia with Staphylococcus aureus after perineal superinfection. Thus, staphylococcus was the principle bacterial species to infect the device and the overall rate of first possible catheter related bacteraemia was $17 \%$ per patient, $15 \%$ /port or $0.05 / 100$ catheter days.

Table 3 shows the risk factors for infection. Neutropenia was not analysed as all children experienced this at least once during treatment. However, intensity of treatment was predictive of infection and this was probably related to the duration of neutropenia. Underlying disease was found not to be significantly related to infection rates. The highest rates of infectious complications occurred in children under 4 of whom all but nine had such complications. Among these younger 39 patients, local infection occurred in 10 compared with two of 141 older children $(p<0.0001)$. Furthermore, most systemic infections with $S$ epidermidis occurred in this subset of patients (20/39) compared with older children (11/141), which is also significant $(p<0.0001)$. The second significant risk factor was the period of use. For reasons we cannot explain, the rate of infection was higher in 1992 with 25 infectious episodes noted in 59 ports compared with 19 in 128 during previous years $(p=0 \cdot 00004)$. Moreover, $S$ epidermidis accounted for the majority of infections during this period, being found in 17 out of 24 patients with documented sepsis: this organism grew from blood cultures in 17 cases in 26 ports inserted in 1992 and eight times in the 88 devices implanted previously $(p<0.0001)$ and was found in 17 out of 59 used subcutaneous ports during $1992 v 12$ out of the 128 used before this period $(p=0 \cdot 0006)$. From February 1993, with the expectation of decreasing this rate of infection, as already reported with external catheters, ${ }^{15}$ we instituted the practice of antibiotic flush with vancomycin. The rate of positive blood cultures with $S$ epidermidis decreased from 26 out of 83 ports ( 32500 catheter days), to four out of 97 (23 270 catheter days) after the initiation of this technique $(p<0.0001)$. Interestingly, the latter four infections occurred in two children who had previously developed septicaemia due to $S$ epidermidis. As shown in the figure, the infection free interval was significantly better after we initiated this technique (log rank test $=0.02$ ).

\section{MECHANICAL COMPLICATIONS}

The incidence of mechanical complications was $10 \cdot 5 \%$. On 17 occasions the catheters became obstructed because of clots $(n=16)$ or chemical reaction between alkaline infusion and calcium $(n=1)$. The lines were cleared in 14 instances with urokinase, but the catheter had to be replaced in three cases. In one obese

Table 3 Risk factors for infection

\begin{tabular}{|c|c|c|}
\hline Characteristic & $\begin{array}{l}\text { Incidence } \\
\text { of infection }\end{array}$ & p Value \\
\hline \multicolumn{3}{|l|}{ Age (years) } \\
\hline $\begin{array}{l}\leq 4 \\
>4\end{array}$ & $\begin{array}{l}30 / 39 \\
36 / 141\end{array}$ & 0.0000001 \\
\hline \multicolumn{3}{|l|}{ Diagnosis } \\
\hline $\begin{array}{l}\text { Acute lymphoblastic leukaemia } \\
\text { Acute non-lymphoblastic }\end{array}$ & $42 / 87$ & \\
\hline $\begin{array}{l}\text { Acute non-lymphoblastic } \\
\text { leukaemia }\end{array}$ & $3 / 7$ & \\
\hline $\begin{array}{l}\text { Non-Hodgkin's lymphoma } \\
\text { Solid tumour }\end{array}$ & $\begin{array}{r}7 / 33 \\
1453\end{array}$ & NS \\
\hline \multicolumn{3}{|l|}{$\begin{array}{l}\text { Solla tumour } \\
\text { Intensity of treatment }\end{array}$} \\
\hline $\begin{array}{l}\text { Moderate } \\
\text { Intensive }\end{array}$ & $2 / 13$ & \\
\hline Very intensive & $28 / 48$ & 0.001 \\
\hline \multicolumn{3}{|l|}{ Period of use } \\
\hline 1988 & $1 / 15$ & \\
\hline 1989 & $7 / 23$ & \\
\hline 1990 & $4 / 35$ & \\
\hline 1991 & $7 / 55$ & \\
\hline 1992 & $25 / 59$ & 0.00004 \\
\hline 1993 & $13 / 73$ & \\
\hline 1994 & $4 / 43$ & \\
\hline \multicolumn{3}{|c|}{ Staphylococcus epidermidis and vancomycin flushing } \\
\hline $\begin{array}{l}\text { Not used } \\
\text { Used }\end{array}$ & $26 / 83$ & 0.0000005 \\
\hline
\end{tabular}

^Not used before February 1993/used after February 1993. 


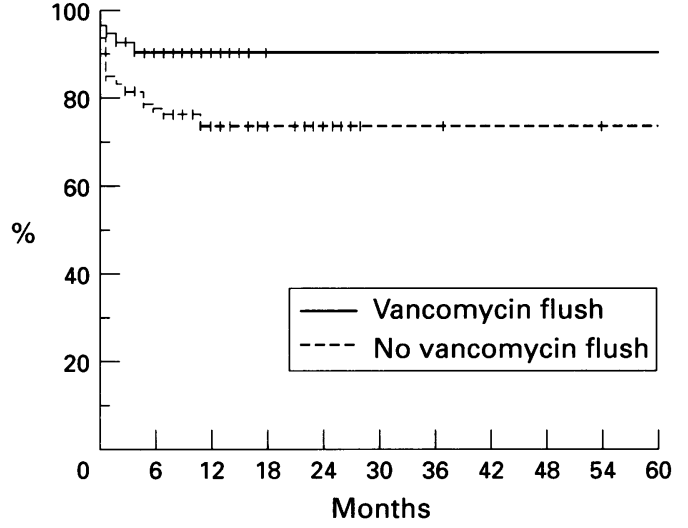

Staphylococcus infection free interval $(n=180)$;

vancomycin flush $(n=64)$, no vancomycin flush $(n=116)$.

child the port had to be replaced because the needle was implanted too deeply so was out of reach. The port blocked in two children due to sharp angulation of the hub at the neck vein entry site related to port dislodgement; this required reoperation. No other mechanical problems, such as clinical evidence of severe venous thrombosis, were encountered.

\section{Discussion}

The use of venous access devices in children with cancer has made it much easier to administer chemotherapy and supportive care. However, infection is one of the main limiting factors for the use of external catheters as its reported incidence varies from $22 \%$ to $88 \%$ or 0.15 to 0.68 episodes $/ 100$ days of catheter use. ${ }^{12}$ Furthermore, the need for frequent maintenance and discomfort for the patient have limited the use of such devices. These are the reasons why totally implanted subcutaneous venous access systems have gained popularity but experience is limited in childhood. Subcutaneous ports were successfully implanted in 163 consecutive patients over 1 year of age who were to receive intensive treatment. External catheters were inserted only in infants or in children who weighed less than $10 \mathrm{~kg}$ because we assumed that their cutaneous barrier would be too frail. With the exception of local haematomas that subsequently resolved, there were no major postoperative complications. All venous access was carried out through the device including blood sampling, chemotherapy, intravenous fluids, blood transfusions, total parenteral nutrition, antibiotics, and other intravenous supportive care. Very intensive treatment

Table 4 Comparison of infection rates with other series

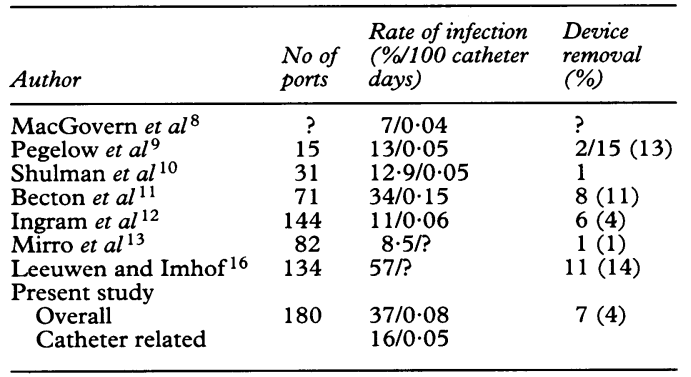

could also be administered through a subcutaneous port as 16 children underwent BMT with their device, as previously reported. ${ }^{16}$

The overall sepsis rate in our series was $37 \%$ or $0 \cdot 12 / 100$ catheter days. Although comparison of infection rates between various published series is complicated by differences in what is defined as a catheter infection, the incidence of infection seems higher in our experience than in other reports. ${ }^{8-1316}$ There are a number of possible explanations. Firstly, we included local infection in addition to positive blood cultures; the former has not been included in other reports. Secondly, blood cultures were obtained exclusively through the system, which might have increased the number of positive strains because of skin contamination. Thirdly, as suggested by the large number of ports used in children receiving intensive chemotherapy $(167 / 180)$, we assume that the duration of neutropenia and immunosuppression was larger in our patients, although the degree of myelosuppression is not clearly described in other reports. Again, five of our second or third infections due to staphylococci and corynebacteria were recurrence of the first septicaemia. Thus, the true incidence of catheter related infection in our series was $15 \%$ or $0.05 / 100$ catheter days. As shown in table 4, these results are comparable with other reported studies. Most infections (55/66) resolved with appropriate antibiotic treatment. We analysed the risk factors for infection. Underlying disease was not a promoting factor, perhaps because malignancies with a good prognosis received light chemotherapy without catheter placement. Most remaining patients had intensive regimens and, although there was a trend for children receiving the most intensive treatment to develop infection, this did not reach significance. Age was a significant factor, as $77 \%$ of children under 4 suffered from an infectious episode. Ingram et al reported an incidence of infection of $12 \%$ or $0.05 / 100$ catheter days in 17 infants under 2 years of age with a subcutaneous port, ${ }^{12}$ which was comparable with the rate found in 127 older patients. As local infections and $S$ epidermidis were the most prominent features of infection in this subset of patients, we suggest that skin fragility and restlessness of such children may increase their risk of infection. On the other hand, we found significantly more staphylococcus infections in 1992 than at other times. This could be related to the unsuitable noncoring needles and Gripper systems which were available at this period, when skin deterioration was noted more often by the nurses. An alternative explanation might be the routine use of transparent dressings which have been reported to increase the incidence of local and systemic infections. ${ }^{17}$ However, we have been using this type of dressing throughout our study in order to closely observe infusions of vesicant chemotherapy, and no change of dressing technique was made in 1992. Because of this rate of superinfection and the uncertainty of its causes, we instituted 
the practice of local antibiotic prophylaxis from February 1993. The antibiotic flush technique was first reported in children with external catheters receiving parenteral nutrition and proved to be efficient in avoiding device removal. ${ }^{18}$ In children with cancer, Schwartz et al reported a prospective randomised study of external catheters, evaluating the efficacy of daily flushes of vancomycin $(25 \mu \mathrm{g} / \mathrm{ml})$ added to heparin, compared with heparin alone. ${ }^{15}$ In the group receiving vancomycin, no catheter related infection was observed and no toxicity was noted. We used higher concentration of vancomycin solution $(50 \mu \mathrm{g} / \mathrm{ml})$ because this had been proved to be stable and efficient even after one month (data not shown), which was mandatory for devices not in use. We confirm the efficacy of such an approach as the staphylococcus sepsis rate dropped from $31 \%$ to $4 \%$. Furthermore, these four infections were observed in two children who had both previously developed septicaemia with $S$ epidermidis; we speculate that sterilisation had not been complete despite appropriate antibiotic treatment. Interestingly, we have not had to remove a device because of infection since we began using this technique.

Mechanical complications occurred in 19 cases $(10.5 \%)$. The catheter clogged in 16 patients and resolved in 14 after instillation of urokinase. Precipitation of substances within the catheter was noted in one case because of the accidental mixture of alkaline infusion and calcium. The low incidence of this complication is probably due to our infrequent use of teniposide.

Neither rupture nor tissue scleroses due to extravasation by chemotherapy was seen. Only four of 19 subcutaneous ports with mechanical complication had to be replaced. This rate is similar to other series in which the reported incidence varies between 5 and $13 \% .{ }^{910}$ Patient and parent acceptance of a subcutaneous port was excellent. Some patients had previously been treated with an external catheter but preferred the port. All interviewees confirmed previously reported observations such as less alteration in body image, less need for heparinisation and dressing changes, and the freedom to shower and bathe. ${ }^{10} 12$ The only reported inconvenience was pain inflicted by the needle placement and related anxiety, especially in younger children. Recently, a topical anaesthetic ointment (Emla, a eutectic mixture of local anaesthetic $5 \%$ ), has proved an efficient and safe procedure to avoid pain associated with port entry. ${ }^{19} 20$ We have used it successfully for all patients since it became available. Frequency of care was reduced by a factor of four compared with external catheters, which has resulted in a saving of approximately 30 minutes/patient/week, or 45 hours/month. From an economic point of view, if the initial cost of the subcutaneous port is higher than the external line ( $£ 155.33$ compared with $£ 88.70$ ), the cumulative cost of maintaining an external line rapidly exceeds the cost of the port.

In conclusion, we confirm that the use of subcutaneous ports in children with cancer is feasible for the majority. Our complication rates are comparable with those reported in other series. The major problem encountered in such high risk patients, that is catheter related infection, can be partly overcome by local antibiotic prophylaxis, although a prospective and randomised study would be needed to confirm this. The technical and psychological advantages of this technique are substantial.

We thank Dr S M Chittal and Dr $\mathrm{H}$ Marcovitch for critical review of the manuscript, Mr A Catteau for his technical assistance, the nursing staff for their rigorous and devoted care, and M F Coutens for help in typing the manuscript.

1 Darbyshire PJ, Weightman NC, Speller DCE. Problems associated with indwelling central venous catheters. Arch Dis Child 1985; 60: 129-34.

2 Cairo MS, Spooner S, Sonder L, et al. Long-term use of indwelling multipurpose silastic catheters in pediatric cancer patients treated with aggressive chemotherapy. f Clin Oncol 1986; 4: 784-8.

3 Sitges-Serra A, Linares J, Garau J. Catheter sepsis: the clue is the hub. Surgery 1985; 97: 355-7.

4 Decker $M D$, Edwards KM. Central venous catheter infections. Pediatr Clin North Am 1988; 35: 579-612.

5 Van Hoff J, Berg AT, Seashore JH. The effect of right atrial catheters on infectious complications of chemotherapy in catheters on infectious complications of

6 Gyves JW, Ensminger WD, Niederhuber JE, et al. A totally implanted injection port system for blood sampling and chemotherapy administration. $\mathcal{F} A M A 1984 ; 25: 2538-41$.

7 Lokich J, Bothe A, Benotti P, et al. Complications and management of implanted venous access catheters. $\mathcal{F}$ Clin Oncol 1985; 3: 710-7.

8 McGovern B, Solenberger R, Reed K. A totally implantable venous access system for long-term chemotherapy in children. $\mathscr{f}$ Pediatr Surg 1985; 20: 725-7.

9 Pegelow CH, Narvaez M, Toledano SR, et al. Experience with a totally implantable venous device in children. Am f Dis Child 1986; 140: 69-71.

10 Shulman RJ, Rahman S, Mahoney D, et al. A totally implanted venous access system used in pediatric patients with cancer. F Clin Oncol 1987; 5: 137-40.

11 Becton DL, Kletzel M, Golladay ES, et al. An experience with an implantable port system in 66 children with cancer. Cancer 1988; 61: 376-8.

12 Ingram J, Weitzmant S, Greenberg ML, et al. Complications of indwelling venous access lines in pediatric hematology patients: a prospective comparison of external venous catheters and subcutaneous ports. Am f Pediatr Hematol Oncol 1991; 13: 130-6.

13 Mirro J, Rao BN, Stokes DC, et al. A prospective study of Hickman/Broviac catheters and implantable ports in pediatric oncology patients. 7 Clin Oncol 1989; 7: 214-22. Elandt-Johnson RC, Johnson NL. Survival models and data analysis. New York: Wiley, 1980: 150-80.

15 Schwartz C, Henrickson KJ, Roghmann K, et al. Prevention of bacteriemia attributed to luminal colonization of tunneled central venous catheters with vancomycin

6 Van Leeuwen EF, Imhof SM. Septicemia in children with cancer and Port-a-Cath. Med Pediatr Oncol 1990; 18 (5): 424 (abstr No 216)

17 Conly JM, Grieves K, Peters B. A prospective randomized study comparing transparent and dry gauze dressings for central venous catheters. F Infect Dis 1989; 159: $310-9$.

18 Messing B, Peitra-Cohen S, Debure A, et al. Antibiotic-lock technique: a new approach to optimal therapy for catheter-related sepsis in home-parenteral nutrition patients. Fournal of Parenteral and Enteral Nutrition 1988; 12: 185-8.

19 Hanks GW, White I. Local anesthesic creams. BMf 1988; 297: 1215-6.

20 Halperin D, Koren G, Attias D, et al. Topical skin anesthesis for venous subcutaneous drug reservoir and lumbar punctures in children. Pediatrics 1989; 84: 281-4. 